\title{
Teleeducación: Brecha digital una realidad palpable: una mirada desde la comunidad educativa
}

\author{
Teleeducation: Digital divide a palpable reality: a look from the educational community
}

Teleeducacion: A divisao digital uma realidade palpavel: um olhar da comunidade educacional

\author{
Milton Hidalgo Achig \\ m.hidalgo@istvicenteleon.edu.ec \\ https://orcid.org/0000-0001-5184-3110 \\ Stalin Salguero Núñez \\ s.salguero@istvicenteleon.edu.ec \\ https://orcid.org/0000-0003-0270-3706
}

Instituto Superior Tecnológico Vicente León, Ecuador

Marco Sandoval Cárdenas

m.sandoval@istvicenteleon.edu.ec

https://orcid.org/0000-0002-0392-2276

Instituto Superior Tecnológico Vicente León, Ecuador
Instituto Superior Tecnológico Vicente León, Ecuador

Juan Iza Garnica

j.iza@istvicenteleon.edu.ec

https://orcid.org/0000-0002-2926-358X

Instituto Superior Tecnológico Vicente León, Ecuador

Artículo recibido 13 de septiembre 2021, arbitrado y aceptado 26 de octubre 2021 y publicado 28 de diciembre 2021

\section{RESUMEN}

Ante la pandemia del Covid-19, las instituciones educativas se han visto obligadas a adoptar los medios y recursos tecnológicos para dar continuidad al proceso enseñanza $\mathrm{y}$ aprendizaje, aunque los beneficios son múltiples, lo cierto es que, ante la desigualdad digital persistente en nuestro medio, ha sido difícil casi imposible aprovechar al máximo estas herramientas, situación que ha truncado la calidad educativa y dificultado la transferencia de conocimientos. La investigación analiza la teleeducación y el impacto que genera la brecha digital en los actores de la comunidad educativa el estudio dio como resultado que los docentes, estudiantes y familias pertenecientes a la educación básica son los más renuentes a adaptarse a ambientes tecnológicos, tienen menor disponibilidad de recursos y un nivel bajo de competencias virtuales, teniendo dificultades para generar espacios virtuales de aprendizaje colaborativo y participativo mientras que los pertenecientes a educación superior no muestran mayor alteración emocional ni funcional se encuentra más familiarizado con plataformas educativas en línea, un factor común en el que coinciden los grupos de estudio es la disminución de la calidad educativa asociada a una mayor carga y estrés laboral.

Palabras clave: Educación; Educación a distancia; Tecnología; Tecnología educacional
ABSTRACT

Faced with the Covid-19 pandemic, educational institutions have been forced to adopt technological means and resources to give continuity to the teaching and learning process, although the benefits are multiple, the truth is that, given the persistent digital inequality in our environment, it has been difficult almost impossible to make the most of these tools, a situation that has truncated educational quality and hampered the transfer of knowledge. This research analyzes teleeducation and the impact generated by the digital divide on the actors of the educational community. The study resulted in that teachers, students and families belonging to basic education are the most reluctant to adapt to technological environments, they have less availability of resources and a low level of virtual skills, having difficulties to generate virtual spaces for collaborative and participatory learning while those belonging to higher education do not show greater emotional or functional alteration is more familiar with online educational platforms, a common factor in the one that the study groups coincide is the decrease in educational quality associated with a greater work load and stress.

Key words: Education; Distance education; Technology; Educational technology

\section{RESUMO}

Diante da pandemia de Covid-19, as instituições de ensino foram obrigadas a adotar meios e recursos tecnológicos para dar continuidade ao processo ensinoaprendizagem, embora os benefícios sejam múltiplos, a verdade é que, dada a persistente desigualdade digital em nosso meio, ela tem sido difícil quase impossível aproveitar ao máximo essas ferramentas, situação que tem truncado a qualidade educacional $\mathrm{e}$ dificultado a transferência de conhecimento. Esta pesquisa analisa a teleducação e o impacto que a exclusão digital gera nos atores da comunidade educacional. $\mathrm{O}$ estudo resultou em que professores, alunos e famílias pertencentes à educação básica são os mais relutantes em se adaptarem aos ambientes tecnológicos, pois têm menor disponibilidade de recursos. E um baixo nível de habilidades virtuais, tendo dificuldade em gerar espaços virtuais de aprendizagem colaborativa e participativa enquanto os pertencentes ao ensino superior não apresentam maior alteração emocional ou funcional está mais familiarizado com as plataformas educacionais online, fator comum naquela que estuda grupos coincidem é a diminuição da qualidade educacional associada a uma maior carga de trabalho e estresse.

Palavras-chave: Educação; Educação a distância; Tecnologia; Tecnologia educacional 


\section{INTRODUCCIÓN}

La rápida innovación tecnológica ha revolucionado todos los campos de la sociedad actual y la educación no ha sido la excepción, es así, que existen sin número de herramientas con características y servicios específicos que permiten desde crear presentaciones y documentos interactivos, hasta aulas completas en línea e interactuar en tiempo real con personas de cualquier parte del mundo, sin duda la conectividad ha dado pasos agigantados, derrumbando barreras de tiempo y distancia. Según la Unir (2021) "la innovación tecnológica en la educación es uno de los principales retos de Universidades y Centros Educativos ya que gracias a estos avances se logrará optimizar y mejorar la experiencia de los estudiantes en el proceso de aprendizaje" (p.12).

Desde que se originó la pandemia del Covid-19, en Ecuador como en el mundo entero se vivieron cambios sin precedentes, los centros educativos no fueron la excepción. Según UNICEF (2020) en el país "el cierre de las escuelas sigue afectando a 4.5 millones de niños, niñas y adolescentes, según una encuesta en línea realizada por UNICEF a estudiantes de 10 a 19 años, el 61,2\% considera que están aprendiendo menos desde el cierre de las escuelas" (p.14).

Al evidenciar que la situación no era pasajera si no una pandemia global que involucraba miles y miles de muertes diarias, las autoridades debían tomar decisiones, plantear alternativas que además de proteger la vida permitan la continuidad económica, social y educativa, es así que decidieron incorporar el teletrabajo y teleeducación como medida de emergencia, sin duda ante la compleja situación sanitaria esta fue una decisión acertada, pero pronto empezaron a surgir inconvenientes derivados de la brecha tecnológica existente en el país. Bonilla (2020) afirma que "la educación mundial y ecuatoriana enfrenta varios retos, de un momento a otro todo el entorno educativo cambió, los decretos de gobierno para detener el brote de propagación determinaron cerrar los establecimientos educativos, marcando una brecha significativa" (p.26).

Lo cierto es que ante la situación del covid-19, todos los aspectos de la vida del ser humano se vieron modificados de uno u otra forma sobre todo con la inmersión de la tecnología en los procesos entre ellos en el sistema educativo, a pesar que las últimas generaciones han crecido apegados a la tecnología y cada vez son más populares las plataformas digitales y el acceso a internet migrar la educación a medios completamente online provocó un retroceso o declive de la calidad educativa (Bedoya y Otros, 2021). Trascender de la presencialidad a la digitalización constituye un reto para el ser humano en todos los ámbitos de la cotidianidad, especialmente en la educación. (Lozada y Aristizabal, 2021).

Ecuador es un país en vías de desarrollo que ya con antelación a la pandemia presentó antecedentes de crisis, considerando a Pérez y Tramallino (2020) se puede afirmar que las estadísticas reflejan que más del 50\% de la población estudiantil especialmente aquellos marginados o poco favorecidos no poseen las herramientas $y / o$ recursos necesarios para acceder a un computador o disponer de internet, incrementando el índice de deserción y abandono escolar, como consecuencia de adoptar decisiones basadas en la urgencia de dar continuidad al proceso de enseñanza en un contexto de crisis sanitaria, 
sin tener en cuenta la calidad y accesibilidad esto perjudica el ejercicio del derecho a la educación libre y gratuita. Un aspecto importante que se debe destacar es el desafío que supone la educación en línea para los padres al tener que ejecutar una educación constructivista en sus hogares y la frustración de estudiantes, padres y profesores al tratar desesperadamente de sostener aprendizajes y vínculos de manera virtual (Castro, 2020).

Aunque la inmersión de las TIC en la educación viene desde años atrás, la teleeducación va más allá del uso básico de un computador o de una pizarra digital, requiere un mayor esfuerzo, en este contexto los padres y la familia ha tomado un papel protagónico en la educación, en concordancia con Carreño (2020) en la fase de aislamiento social, en Ecuador la teleeducación han resaltado situaciones críticas para la educación como problemas de conectividad, la falta de herramientas tecnológicas en los hogares, la deserción y abandono escolar, todo esto deja bajas en la calidad en la educación, sobretodo el acceso a la educación en la zonas rurales y marginadas es ínfimo, esto impide que miles de niñas, niños y adolescentes puedan continuar con sus estudios incrementando el índice de analfabetismo, pobreza, mendicidad y trabajo infantil.

Los estudiantes identifican como las principales dificultades asociadas al cambio de modalidad, en orden de relevancia en cantidad de respuestas: la afectación emocional, la sobrecarga de actividades de enseñanza, la falta de contacto con docentes y compañeros, las dificultades en el acceso a la bibliografía. (Ramos y Otros, 2021, p.6)

Las preguntas clave que se derivan de la situación actual de Ecuador es ¿en realidad funciona la teleeducación?, ¿Con la nueva modalidad se está logrando cumplir los objetivos educativos?, ¿Los docentes, estudiantes y familias están realmente preparados para afrontar la educación totalmente en línea?, ¿Es realmente una experiencia propicia para los niños, adolescentes y sus familias?, para abordar el problema en sí, es necesario efectuar un análisis un poco más profundo por esta razón el presente artículo se orienta a analizar la educación a nivel inicial, básica y superior, descomponiendo la comunidad educativa en función a tres aspectos los docentes, los estudiantes y las familias para analizar desde una visión panorámica las características y capacidades tecnológicas individuales de cada grupo y como se relacionan e interactúan en el proceso educativo, con el fin de establecer el grado de desigualdad tecnológica y el impacto que genera en la calidad de transmisión y recepción de conocimientos, en un país donde la realidad palpable es desfavorable para ciertos sectores, el acceso a internet es limitado y de baja calidad, es necesario identificar las consecuencias del cambio de modalidad en educación y definir si realmente se ha logrado dar continuidad al proceso de enseñanza logrando un aprendizaje holístico o solo se ha procurado no perder un periodo académico y los resultados en enseñanza aprendizaje dejan mucho que desear.

\section{MÉTODO}

El estudio se orientó a una metodología transversal de tipo descriptivo interpretativo, como instrumento de recolección de datos se empleó la encuesta mediante cuestionarios previamente elaborados, la recolección de datos se efectuó en un lapso de tiempo específico que corresponde al 
periodo enero-diciembre 2020, la población de estudio se obtuvo con datos de fuentes oficiales como la Dirección Distrital de Educación 05D01 Latacunga y la Secretaría de Educación Superior, Ciencia, Tecnología e innovación, la muestra fue calculada con un margen de error aceptable del $5 \%$ y una seguridad razonable del 95\%, el alcance de la investigación se extiende a tres dimensiones docentes, estudiantes y familias de las comunidades educativas de educación básica, media y superior.

La población total estuvo compuesta por estudiantes, docentes y familias que pertenecen a la educación básica, media y superior de instituciones públicas y privadas del cantón Latacunga, provincia de Cotopaxi.

Teniendo presente las características de la población los niños y adolescentes presentan una mayor dependencia de ayuda por parte de los padres o algún familiar cercano para la ejecución de tareas, proyectos y actividades en relación a los jóvenes de las instituciones superiores como institutos o universidades, para facilitar la aplicación de instrumentos, se dividió la población en dos grandes grupos la educación básica y media Grupo A y la educación superior Grupo B.

Según la Dirección Distrital 05D01 Latacunga (2020) el grupo A (Tabla 1) cuenta con 120 Instituciones Educativas repartidos en los 12 circuitos conformados por 79 instituciones fiscales, 5 instituciones fiscomisionales y 36 instituciones particulares, atendiendo a 57.284 estudiantes en los diferentes niveles educativos, desde Educación Inicial Subnivel 2, Educación General Básica, Bachillerato General Unificado en Ciencias, Bachillerato General Unificado Técnico y Bachillerato Internacional (Tabla 2) (pp.9-11).

Tabla 1. Grupo A - Educación Básica y Media.

\begin{tabular}{|c|c|c|}
\hline COBERTURA & PARCIAL & DATOS \\
\hline Población - Cantón & & 202.878 \\
\hline Parroquias & & 15 \\
\hline Urbanas & 5 & \\
\hline Rurales & 10 & \\
\hline Circuitos & & 12 \\
\hline Población Estudiantil & & 57.284 \\
\hline Establecimientos Educativos & & 120 \\
\hline Fiscales & 79 & \\
\hline Fiscomisionales & 5 & \\
\hline Particulares & 36 & \\
\hline
\end{tabular}

Nota: Elaboración propia de los autores, fuente Dirección Distrital 05D01 (2020). 
Tabla 2. Instituciones por Circuitos.

\begin{tabular}{cllc}
\hline $\mathbf{N}^{\circ}$ & \multicolumn{1}{c}{ CÓDIGO } & \multicolumn{1}{c}{ NOMBRE DEL CIRCUITO } & DATOS \\
\hline 1 & 05D01C01 & Toacaso & 7 \\
2 & 05D01C02 & Mulaló & 4 \\
3 & 05D01C03_18 & Guaytacama- Tanicuchi & 8 \\
4 & 05D01C04 & Aláquez - José Guango Bajo & 3 \\
5 & 05D01C05 & Once de Noviembre - Poaló & 14 \\
6 & 05D01C06_11 & San Buena Ventura - Juan Montalvo & 10 \\
7 & 05D01C07_12 & Eloy Alfaro (San Felipe) & 8 \\
8 & 05D01C08_13 & Eloy Alfaro - Ignacio Flores (Parque Flores) & 8 \\
9 & 05D01C09_10 & Ignacio Flores - Juan Montalvo (San Sebastián) & 14 \\
10 & 05D01C14_15 & La Matriz & 32 \\
11 & 05D01C16 & Belisario Quevedo (Guanailin) & 4 \\
12 & 05D01C17 S & San Juan De Pastocalle & 8 \\
\hline
\end{tabular}

Nota: Elaboración propia de los autores, fuente Dirección Distrital 05D01 (2020).

El grupo B educación superior (Tabla 3) es un tanto más reducido existen en Latacunga dos universidades Universidad Técnica de Cotopaxi y La Universidad de las Fuerzas Armadas ESPE, en cuánto a Institutos Tecnológicos existen tres Instituto Superior Técnico Vicente León, Instituto
Superior Victoria Vascones Cuvi y el Instituto Territorial Cotopaxi.

Según SENESCYT (2020) “existen 9.800 estudiantes en Universidades y Escuelas Politécnicas y 2.833 en Institutos Técnicos y Tecnológicos" (P.54).

Tabla 3. Grupo B Educación Superior.

\begin{tabular}{lc}
\hline \multicolumn{1}{c}{ INSTITUCIONES } & DATOS \\
\hline Universidades y Escuelas Politécnicas & 9.800 \\
Institutos Superiores Técnicos y Tecnológicos & 2.833 \\
\hline Total de estudiantes & $\mathbf{1 2 . 6 3 3}$
\end{tabular}

Nota: Elaboración propia de los autores, fuente Secretaría de Educación Superior, Ciencia y Tecnología (SENESCYT), mediante el Sistema Nacional de Información de Educación Superior Ecuador (SNIESE) con corte del año (2020).

La población total presente en la Tabla 4 de la investigación se muestra de la siguiente manera:

Tabla 4. Población total.

\begin{tabular}{ll}
\hline \multicolumn{1}{c}{ POBLACIÓN } & TOTAL \\
\hline Grupo A & 57.284 \\
Grupo B & 12.633 \\
\hline POBLACIÓN TOTAL & $\mathbf{6 9 . 9 1 7}$ \\
\hline
\end{tabular}

Nota: Elaboración propia de los autores. 
La muestra se obtuvo de la siguiente manera:

$$
\begin{gathered}
\mathrm{n}=\frac{\mathrm{N} * \mathrm{Z}^{2} * \mathrm{p} * \mathrm{q}}{\mathrm{E}^{2}(\mathrm{~N}-1)+\mathrm{Z}^{2} * \mathrm{p} * \mathrm{q}} \\
\mathrm{n}=\frac{69.917 * 1,96^{2} * 0,5 * 0,5}{0,05^{2}(69.917-1)+1,96^{2} * 0,50 * 0,50}=\frac{67.148,2868}{175,7504}=382,06=382
\end{gathered}
$$
n: Muestra
E: Margen de error 5\%: 0,05
q: Nivel de rechazo aceptable 50\%: 0,5
p: Nivel de aceptación aceptable 50\%: 0,5
Z: Desviación clásica: 95\%: 1,96
N: Población total

La distribución de la muestra se aprecia en la Tabla 5.

Tabla 5. Muestra por Grupos.

\begin{tabular}{lll}
\hline & Muestra Total & $\mathbf{3 8 2}$ \\
\hline Grupo 1 & 267 \\
Grupo 2 & 115 \\
\hline
\end{tabular}

Nota: Elaboración propia de los autores.

Docentes Grupo 1. En los docentes de escuelas, colegios yunidades educativas deldistrito Latacunga, con una muestra representativa se logró identificar que el $70 \%$ disponía de un computador que usaba para preparar los instrumentos pedagógicos antes de la pandemia; solo el 23\% disponía de conectividad a internet en sus hogares, el uso de redes sociales es de hasta un $84 \%$, los programas básicos como Word, Excel, etc. era conocido por el 95\%, estos les permitían preparar archivos y documentos para las clases presenciales pero, el $93 \%$ de docentes no había preparado ni impartido una clase virtual antes de la emergencia; esto refleja la experiencia que viene transitando el quehacer docente en tiempos de pandemia mediante la adopción acelerada y forzada del uso de herramientas, programas y aplicaciones en línea como Meet, zoom y el mismo Microsoft Teams, en general una forma de trabajo totalmente nueva y desconocida, el $98 \%$ refleja haber tenido inconvenientes para adaptarse a la nueva modalidad, aunque les brindaron capacitaciones el $79 \%$ presentó problemas en su proceso de enseñanza; en la actualidad al 71\% le resulta difícil el manejo de estos instrumentos, al 60\% le tomó por lo menos un año aprender a usar estos medios, esto puede deberse a que un gran porcentaje de docentes presenta una edad avanzada, por lo tanto el uso de un computador, de plataformas y aplicaciones resulta un tanto complejo. Sin duda esto repercute en la calidad de la educación un $80 \%$ concuerda que, si bien ya existían fallos en la educación presencial a nivel país, con la teleeducación la situación es más crítica, pues para el docente representa un mayor nivel de estrés, cansancio físico y psicológico, por la presión que genera tener que adaptarse a un ambiente totalmente en línea (Tabla 6). 
Docentes Grupo 2. En la investigación realizada es evidente que los docentes de las IES presentan una mayor familiaridad con las plataformas digitales y se les es más fácil adaptarse a la nueva tecnología, esto puede deberse a que la labor docente en instituciones de educación superior está regida por tres ejes sustantivos investigación, vinculación y la docencia en sí, aunque siguen teniendo las actividades propias de la enseñanza como preparar e impartir clases, evaluar y calificar trabajos, reunirse en tiempo real con compañeros y estudiantes, para ellos demanda un mayor esfuerzo, programas investigativos vinculados a la sociedad y emplean en el quehacer diario tareas, pruebas digitalizadas; por esta razón les causa un impacto menor el cambio de modalidad. De la totalidad de docentes encuestados el 95\% disponía de un computador antes de la pandemia y el $73 \%$ contaba con conectividad en su hogar y aunque el $89 \%$ no había impartido propiamente una clase de forma virtual, luego de las capacitaciones recibidas al menos el $50 \%$ pudo manejar las aplicaciones sin mayor problema, a la mayoría de docentes les tomó hasta seis meses aprender el uso de los medios tecnológicos a diferencia de los docentes del grupo 1 que al menos al 60\% le tomó un año y al 35\% más de un año, este es un aspecto importante puesto que se aprecia una mayor capacidad de adaptación al cambio por parte de docentes universitarios y una mayor complejidad al uso de tecnología en docentes de nivel básico - medio; en cuánto a la calidad educativa tanto el grupo 1 y 2 concuerdan en mayoría que es menor en modalidad virtual y que el estrés y sobrecarga laboral incrementa con el teletrabajo, trabajar en el hogar puede resultar un poco más estresante porque el entorno se vuelve complejo al tener que cumplir con sus labores de docente, de madre/padre, ama de casa/jefe de hogar incluso también maestra/o de sus hijos, esto puede desencadenar cansancio físico y psicológico que puede afectar el desempeño, estado de ánimo, mental y de salud. Por último, un aspecto igualmente importante es que los dos grupos concuerdan que el cumplimiento satisfactorio de tareas, la asistencia y puntualidad y el promedio de calificaciones ha disminuido en la teleeducación ocasionando un mayor índice de ausentismo y deserción escolar este es un problema grave, cada vez que un niño se aparta del sistema educativo, se incrementa el analfabetismo, el trabajo infantil y mendicidad. (Tabla 6).

Es importante que las autoridades presten atención a la existencia de estas brechas y las consecuencias que genera, es verdad que no se puede regresar el tiempo y enmendar los errores cometidos, pero es tiempo de ver hacia el futuro procurando que en la nueva realidad como es el ambiente virtual, se introduzca de forma adecuada las Tics en la educación logrando hacer realidad la compleja interrelación y conexión que debe existir entre la tecnología, la pedagogía y el contenido cognitivo de cada asignatura; sin duda si a un docente no cuenta con el conocimiento y capacitación adecuada, se le dificultará la preparación de material didáctico y repercute en la calidad educativa percibida por sus estudiantes. No, se debe pasar por alto los tres aspectos fundamentales en la teleeducación: la conectividad, misma que se percibe como calidad o capacidad de red, la transferencia de información y la comunicación, el uso de la tecnología debe facilitar y agilizar estos dos últimos puntos al romper barreras de tiempo y distancia; no por el contrario entorpecer la transmisión del conocimiento, ni generar cargas innecesarias de presión sobre los usuarios, solo se logrará obtener el mayor beneficio cuando se incremente el conocimiento y los recursos invertidos, puesto que cada día se requiere de nuevos equipos, con funciones mejoradas y servicios más complejos. 
Tabla 6. Resultado Docentes Grupo 1 y 2.

\begin{tabular}{|c|c|c|c|c|c|c|c|c|c|c|c|c|}
\hline \multirow{3}{*}{$\begin{array}{cc}\text { Docentes } \\
\text { Pregunta } \\
\text { Disponía de un computador antes de la pandemia }\end{array}$} & \multicolumn{6}{|c|}{ Grupo 1} & \multicolumn{6}{|c|}{ Grupo 2} \\
\hline & \multicolumn{2}{|c|}{ Si } & \multicolumn{2}{|c|}{ No } & \multicolumn{2}{|c|}{ No Aplica } & \multicolumn{2}{|c|}{ Si } & \multicolumn{2}{|c|}{ No } & \multicolumn{2}{|c|}{ No Aplica } \\
\hline & 187 & $70 \%$ & 80 & $30 \%$ & 0 & $0 \%$ & 109 & $95 \%$ & 6 & $5 \%$ & 0 & $0 \%$ \\
\hline Disponibilidad de conectividad en su hogar antes del confinamiento & 61 & $23 \%$ & 206 & $77 \%$ & 0 & $0 \%$ & 84 & $73 \%$ & 31 & $27 \%$ & 0 & $0 \%$ \\
\hline Usaba redes sociales antes de la pandemia & 224 & $84 \%$ & 43 & $16 \%$ & 0 & $0 \%$ & 106,95 & $93 \%$ & 8 & $7 \%$ & 0 & $0 \%$ \\
\hline Conocía programas informáticos básicos como Word, Excel, PowerPoint & 254 & $95 \%$ & 13 & $5 \%$ & 0 & $0 \%$ & 113 & $98 \%$ & 2 & $2 \%$ & 0 & $0 \%$ \\
\hline Había preparado e impartido clases virtuales antes de la pandemia & 8 & $3 \%$ & 248 & $93 \%$ & 0 & $0 \%$ & 13 & $11 \%$ & 102 & $89 \%$ & 0 & $0 \%$ \\
\hline Tenía conocimiento del manejo de herramientas educativas on-line & 27 & $10 \%$ & 240 & $90 \%$ & 0 & $0 \%$ & 67 & $58 \%$ & 48 & $42 \%$ & 0 & $0 \%$ \\
\hline Conocía Meet, Zoom, etc. & 8 & $3 \%$ & 259 & $97 \%$ & 0 & $0 \%$ & 23 & $20 \%$ & 92 & $80 \%$ & 0 & $0 \%$ \\
\hline Le fue fácil adaptarse a clases virtuales & 5 & $2 \%$ & 262 & $98 \%$ & 0 & $0 \%$ & 72 & $63 \%$ & 43 & $37 \%$ & 0 & $0 \%$ \\
\hline Les brindaron capacitaciones sobre la teleeducación & 227 & $85 \%$ & 40 & $15 \%$ & 0 & $0 \%$ & 112 & $97 \%$ & 3 & $3 \%$ & 0 & $0 \%$ \\
\hline \multirow[t]{2}{*}{ Luego de la capacitación pudo usar las herramientas sin problema } & 56 & $21 \%$ & 211 & $79 \%$ & 0 & $0 \%$ & 57,5 & $50 \%$ & 57,5 & $50 \%$ & 0 & $0 \%$ \\
\hline & \multicolumn{2}{|c|}{ Fácil } & \multicolumn{2}{|c|}{ Medio } & \multicolumn{2}{|c|}{ Difícil } & \multicolumn{2}{|c|}{ Fácil } & \multicolumn{2}{|c|}{ Medio } & \multicolumn{2}{|c|}{ Difícil } \\
\hline \multirow[t]{2}{*}{ Manejar plataformas digitales le resulta } & 3 & $1 \%$ & 75 & $28 \%$ & 190 & $71 \%$ & 48 & $18 \%$ & 112 & $42 \%$ & 107 & $40 \%$ \\
\hline & \multicolumn{2}{|c|}{1 a 6 meses } & \multicolumn{2}{|c|}{1 año } & \multicolumn{2}{|c|}{ (+) 1 año } & \multicolumn{2}{|c|}{1 a 6 meses } & \multicolumn{2}{|c|}{1 año } & \multicolumn{2}{|c|}{ (+) 1 año } \\
\hline Cuánto tiempo le tomó aprender el uso de los medios tecnológicos & 13 & $5 \%$ & 160 & $60 \%$ & 93 & $35 \%$ & 112 & $42 \%$ & 83 & $31 \%$ & 72 & $27 \%$ \\
\hline
\end{tabular}

Horizontes. Revista de Investigación en Ciencia de la Educación 


\begin{tabular}{|c|c|c|c|c|c|c|c|c|c|c|c|c|}
\hline \multirow{4}{*}{$\begin{array}{c}\text { Docentes } \\
\text { Pregunta } \\
\text { La calidad de la educación virtual en referencia con la presencial }\end{array}$} & \multicolumn{6}{|c|}{ Grupo 1} & \multicolumn{6}{|c|}{ Grupo 2} \\
\hline & \multicolumn{2}{|c|}{ Si } & \multicolumn{2}{|c|}{ No } & \multicolumn{2}{|c|}{ No Aplica } & \multicolumn{2}{|c|}{$\mathrm{Si}$} & \multicolumn{2}{|c|}{ No } & \multicolumn{2}{|c|}{ No Aplica } \\
\hline & \multicolumn{2}{|c|}{ Menor } & \multicolumn{2}{|c|}{ Igual } & \multicolumn{2}{|c|}{ Mayor } & \multicolumn{2}{|c|}{ Menor } & \multicolumn{2}{|c|}{ Igual } & \multicolumn{2}{|c|}{ Mayor } \\
\hline & 214 & $80 \%$ & 35 & $13 \%$ & 19 & $7 \%$ & 182 & $68 \%$ & 53 & $20 \%$ & 32 & $12 \%$ \\
\hline El estrés laboral y sobrecarga en teleeducación es & 21 & $8 \%$ & 13 & $5 \%$ & 232 & $87 \%$ & 40 & $15 \%$ & 59 & $22 \%$ & 168 & $63 \%$ \\
\hline La asistencia y puntualidad del alumno & 187 & $70 \%$ & 67 & $25 \%$ & 13 & $5 \%$ & 115 & $43 \%$ & 104 & $39 \%$ & 48 & $18 \%$ \\
\hline Cumplimiento de tareas satisfactorio & 246 & $92 \%$ & 13 & $5 \%$ & 8 & $3 \%$ & 227 & $85 \%$ & 37 & $14 \%$ & 3 & $1 \%$ \\
\hline El promedio de calificaciones & 232 & $87 \%$ & 24 & $9 \%$ & 11 & $4 \%$ & 224 & $84 \%$ & 40 & $15 \%$ & 3 & $1 \%$ \\
\hline Ausentismo escolar & 13 & $5 \%$ & 11 & $4 \%$ & 243 & $91 \%$ & 11 & $4 \%$ & 112 & $42 \%$ & 144 & $54 \%$ \\
\hline \multirow[t]{2}{*}{ Deserción escolar } & 11 & $4 \%$ & 16 & $6 \%$ & 240 & $90 \%$ & 27 & $10 \%$ & 56 & $21 \%$ & 184 & $69 \%$ \\
\hline & \multicolumn{2}{|c|}{ Armonioso } & \multicolumn{2}{|c|}{ Pesado } & \multicolumn{2}{|c|}{ Insostenible } & \multicolumn{2}{|c|}{ Armonioso } & \multicolumn{2}{|c|}{ Pesado } & \multicolumn{2}{|c|}{ Insostenible } \\
\hline El ambiente en su hogar con clases virtuales y teletrabajo le resulta & 21 & $8 \%$ & 85 & $32 \%$ & 160 & $60 \%$ & 45 & $17 \%$ & 112 & $42 \%$ & 109 & $41 \%$ \\
\hline
\end{tabular}

Nota: Elaboración propia de los autores. 
Estudiantes Grupo 1. En el grupo 1 de estudiantes que pertenecen a educación básica y media, la realidad es compleja, la brecha digitaly desigualdades socioeconómicas se hacen presente, antes de la pandemia el $92 \%$ de ellos no contaba con un computador y el 79\% no tenía conexión en su hogar y solo el 50\% manejaba los programas básicos como Word, Excel, y no habían recibido una clase virtual antes de la emergencia sanitaria, se puede afirmar que conocieron por primera vez la plataforma Microsoft Teams en la fase de confinamiento social, por esta razón no les fue fácil adaptarse a la nueva realidad, hasta la actualidad al menos el 30\% de ellos acepta no poder asistir puntualmente a las clases programadas, el $77 \%$ le resulta difícil el manejo de las plataformas, el $32 \%$ posee dispositivos propios para la conexión de clases, mientras que el $48 \%$ utiliza dispositivos prestados y el $20 \%$ no dispone de ningún dispositivo, esta puede ser una razón por la que existe mayor ausentismo y deserción pues al no contar con los medios se les hace imposible asistir a las clases, reuniones y cumplir con las tareas programadas, de igual manera los estudiantes coinciden que la calidad educativa es menor en modalidad teletrabajo y el $49 \%$ afirma haber pensado dejar los estudios en algún momento de la emergencia sanitaria, el 39\% por falta de recursos y el $19 \%$ por las dificultades que representaba el uso de medios tecnológicos virtuales. (Tabla 7).

Estudiantes Grupo 2. Los estudiantes del Grupo 2 corresponde a jóvenes de educación superior, ellos presentan una mayor familiaridad en cuanto al uso de tecnologías y medios o-line, por el uso de redes sociales, asemejan ese tipo de comunicación en lo académico, por esta razón no representa un problema mayor, pues ellos manejan en la vida cotidiana entornos virtuales de comunicación social. Al menos el 58\% disponía de un computador antes de la pandemia y aunque el 90\% no había recibido una clase completamente virtual, los estudiantes mantienen una comunicación activa con los docentes en grupos de redes sociales o correo electrónico, por lo que el 50\% dice haberse adaptado con facilidad al entorno virtual de aprendizaje y el $60 \%$ afirma asistir puntualmente a las clases programadas, el $51 \%$ cuenta con dispositivos propios, sin embargo también afirman percibir una menor calidad educativa en teleeducación y el 50\% acepta haber bajado su rendimiento académico y el 28\% manifiesta haber tenido la idea de dejar sus estudios por falta de recursos económicos y dificultades tecnológicas. (Tabla 7).

Es importante tener en cuenta que la decisión de dejar los estudios en muchas ocasiones se debe a la difícil situación económica que pasan las familias, en este contexto el haber perdido el trabajo o tener que cerrar los negocios pudo contraer una situación crítica en la que debieron buscar alguna fuente de ingreso alternativa y dejar los estudios para ayudar a su familia; si relacionamos los estudiantes del grupo 1 y grupo 2, la diferencia es evidente, las IES tanto en docentes como en estudiantes presenta una mayor familiaridad y fácil adaptación a los ambientes virtuales mientras que para los estudiantes de básica y media resulta sumamente difícil estudiar en modalidad $100 \%$ virtual desde sus hogares por no contar con los medios o por lo complicado que puede ser manejar las plataformas, programas y herramientas; cabe recalcar que las encuestas fueron aplicadas en todos los circuitos que comprende el distrito Latacunga, incluidos aquellos sectores más desfavorecidos, por ello los resultados muestran una dura realidad que acontece en nuestro medio 
Tabla 7. Resultado Estudiantes Grupo 1 y 2.

\begin{tabular}{|c|c|c|c|c|c|c|c|c|c|c|c|c|}
\hline \multirow{3}{*}{$\begin{array}{c}\text { Estudiantes } \\
\text { Pregunta } \\
\text { Disponía de un computador antes de la pandemia }\end{array}$} & \multicolumn{6}{|c|}{ Grupo 1} & \multicolumn{6}{|c|}{ Grupo 2} \\
\hline & \multicolumn{2}{|c|}{ Si } & \multicolumn{2}{|c|}{ No } & \multicolumn{2}{|c|}{ No Aplica } & \multicolumn{2}{|c|}{ Si } & \multicolumn{2}{|c|}{ No } & \multicolumn{2}{|c|}{ No Aplica } \\
\hline & 21 & $8 \%$ & 246 & $92 \%$ & 0 & $0 \%$ & 155 & $58 \%$ & 112 & $42 \%$ & 0 & $0 \%$ \\
\hline Disponibilidad de conectividad en su hogar antes del confinamiento & 56 & $21 \%$ & 211 & $79 \%$ & 0 & $0 \%$ & 139 & $52 \%$ & 128 & $48 \%$ & 0 & $0 \%$ \\
\hline Usaba redes sociales antes de la pandemia & 160 & $60 \%$ & 107 & $40 \%$ & 0 & $0 \%$ & 256 & $96 \%$ & 11 & $4 \%$ & 0 & $0 \%$ \\
\hline Conocía programas informáticos básicos como Word, Excel, PowerPoint & 134 & $50 \%$ & 134 & $50 \%$ & 0 & $0 \%$ & 262 & $98 \%$ & 5 & $2 \%$ & 0 & $0 \%$ \\
\hline Había recibido clases virtuales antes de la pandemia & 0 & $0 \%$ & 267 & $100 \%$ & 0 & $0 \%$ & 27 & $10 \%$ & 240 & $90 \%$ & 0 & $0 \%$ \\
\hline Tenía conocimiento del manejo de herramientas educativas on-line & 0 & $0 \%$ & 267 & $100 \%$ & 0 & $0 \%$ & 107 & $40 \%$ & 160 & $60 \%$ & 0 & $0 \%$ \\
\hline Conocía Meet, Zoom, etc. & 0 & $0 \%$ & 267 & $100 \%$ & 0 & $0 \%$ & 53 & $20 \%$ & 214 & $80 \%$ & 0 & $0 \%$ \\
\hline Le fue fácil adaptarse a clases virtuales & 27 & $10 \%$ & 240 & $90 \%$ & 0 & $0 \%$ & 134 & $50 \%$ & 134 & $50 \%$ & 0 & $0 \%$ \\
\hline Les brindaron capacitaciones sobre la teleeducación & 107 & $40 \%$ & 160 & $60 \%$ & 0 & $0 \%$ & 128 & $48 \%$ & 139 & $52 \%$ & 0 & $0 \%$ \\
\hline \multirow[t]{2}{*}{ Asiste puntualmente a todas las clases virtuales programadas } & 80 & $30 \%$ & 187 & $70 \%$ & 0 & $0 \%$ & 160 & $60 \%$ & 107 & $40 \%$ & 0 & $0 \%$ \\
\hline & \multicolumn{2}{|c|}{ Fácil } & \multicolumn{2}{|c|}{ Medio } & \multicolumn{2}{|c|}{ Difícil } & \multicolumn{2}{|c|}{ Fácil } & \multicolumn{2}{|c|}{ Medio } & \multicolumn{2}{|c|}{ Difícil } \\
\hline \multirow[t]{2}{*}{ Manejar las plataformas digitales le resulta } & 8 & $3 \%$ & 53 & $20 \%$ & 206 & $77 \%$ & 101 & $38 \%$ & 120 & $45 \%$ & 45 & $17 \%$ \\
\hline & \multicolumn{2}{|c|}{1 a 6 meses } & \multicolumn{2}{|c|}{1 año } & \multicolumn{2}{|c|}{ (+) 1 año } & \multicolumn{2}{|c|}{1 a 6 meses } & \multicolumn{2}{|c|}{1 año } & \multicolumn{2}{|c|}{ (+) 1 año } \\
\hline Cuánto tiempo le tomó aprender el uso de los medios tecnológicos & 27 & $10 \%$ & 160 & $60 \%$ & 80 & $30 \%$ & 134 & $50 \%$ & 107 & $40 \%$ & 27 & $10 \%$ \\
\hline
\end{tabular}




\begin{tabular}{|c|c|c|c|c|c|c|c|c|c|c|c|c|}
\hline \multirow{4}{*}{$\begin{array}{c}\text { Estudiantes } \\
\text { Pregunta } \\
\text { Los dispositivos con que se conecta a clases son }\end{array}$} & \multicolumn{6}{|c|}{ Grupo 1} & \multicolumn{6}{|c|}{ Grupo 2} \\
\hline & \multicolumn{2}{|c|}{ Si } & \multicolumn{2}{|c|}{ No } & \multicolumn{2}{|c|}{ No Aplica } & \multicolumn{2}{|c|}{ Si } & \multicolumn{2}{|c|}{ No } & \multicolumn{2}{|c|}{ No Aplica } \\
\hline & \multicolumn{2}{|c|}{ Propios } & \multicolumn{2}{|c|}{ Prestados } & \multicolumn{2}{|c|}{ No dispongo } & \multicolumn{2}{|c|}{ Propios } & \multicolumn{2}{|c|}{ Prestados } & \multicolumn{2}{|c|}{ No dispongo } \\
\hline & 85 & $32 \%$ & 128 & $48 \%$ & 53 & $20 \%$ & 136 & $51 \%$ & 101 & $38 \%$ & 29 & $11 \%$ \\
\hline & \multicolumn{2}{|c|}{ Igual } & \multicolumn{2}{|c|}{ Mayor } & \multicolumn{2}{|c|}{ Menor } & \multicolumn{2}{|c|}{ Igual } & \multicolumn{2}{|c|}{ Mayor } & \multicolumn{2}{|c|}{ Menor } \\
\hline La calidad de la educación virtual en referencia con la presencial & 80 & $30 \%$ & 16 & $6 \%$ & 171 & $64 \%$ & 85 & $32 \%$ & 24 & $9 \%$ & 158 & $59 \%$ \\
\hline \multirow[t]{2}{*}{ Sus calificaciones en este tiempo de pandemia } & 37 & $14 \%$ & 13 & $5 \%$ & 190 & $71 \%$ & 83 & $31 \%$ & 51 & $19 \%$ & 134 & $50 \%$ \\
\hline & \multicolumn{2}{|c|}{ Sí } & \multicolumn{2}{|c|}{ No } & \multicolumn{2}{|c|}{ No Aplica } & \multicolumn{2}{|c|}{ Sí } & \multicolumn{2}{|c|}{ No } & \multicolumn{2}{|c|}{ No Aplica } \\
\hline \multirow[t]{2}{*}{ Ha querido dejar sus estudios en este tiempo de pandemia } & 131 & $49 \%$ & 136 & $51 \%$ & 0 & $0 \%$ & 75 & $28 \%$ & 179 & $67 \%$ & 13 & $5 \%$ \\
\hline & \multicolumn{2}{|c|}{ Falta de recursos } & \multicolumn{2}{|c|}{$\begin{array}{l}\text { Dificultades } \\
\text { tecnológicas }\end{array}$} & \multicolumn{2}{|c|}{ No Aplica } & \multicolumn{2}{|c|}{$\begin{array}{l}\text { Falta de } \\
\text { recursos }\end{array}$} & \multicolumn{2}{|c|}{$\begin{array}{l}\text { Dificultades } \\
\text { tecnológicas }\end{array}$} & \multicolumn{2}{|c|}{ No Aplica } \\
\hline \multirow[t]{2}{*}{ Porque ha querido dejar sus estudios } & 80 & $30 \%$ & 51 & $19 \%$ & 136 & $51 \%$ & 48 & $18 \%$ & 27 & $10 \%$ & 192 & $72 \%$ \\
\hline & & oso & & & & enible & Arn & & & & Inso & iible \\
\hline El ambiente en su hogar con clases virtuales y teletrabajo le resulta & 21 & $8 \%$ & 112 & $42 \%$ & 134 & $50 \%$ & 67 & $25 \%$ & 134 & $50 \%$ & 67 & $25 \%$ \\
\hline
\end{tabular}

Nota: Elaboración propia de los autores. 
Familias Grupo 1. En muchas familias especialmente con estudiantes de educación básica y media, se ha generado un contexto complejo, esto debido a que estos estudiantes presentan una mayor dependencia hacia sus padres o hermanos mayores para realizar sus tareas, por lo tanto requiere un mayor control, esto se dificulta al ser clases totalmente en línea, tener que subir tareas y trabajos a plataformas, los padres que según la encuesta el $81 \%$ desconoce el manejo y uso de este tipo de ambientes virtuales, les es imposible estar al día y tener conocimiento pleno de las actividades que deben realizar sus hijos, ocasionando que los niños y adolescentes oculten información, se despreocupen de sus obligaciones, falten a clases y al final obtengan bajo rendimiento, el $30 \%$ de familias dice desconocer las notas y situación real de sus hijos en la escuela, a esto le sumamos que solo el $20 \%$ disponía de al menos un computador antes de la pandemia, cuando más de un integrante estudia el no contar con los dispositivos necesarios o la conectividad adecuada, puede convertirse en un completo martirio, es así al punto que el $67 \%$ de familias afirma que el ambiente en el hogar se torna insoportable, esto puede influir de forma directa en el comportamiento, rendimiento y bienestar físico y psicológico de todos los integrantes de la familia. (Tabla 8).

Familias Grupo 2. En las familias con estudiantes del nivel superior es un contexto diferente, esto puede referirse al hecho que los jóvenes son más independientes, se representan a sí mismos, tienen una personalidad más centrada y son responsables de sus tareas y rendimiento, los padres no requieren estar presentes en todo momento. El 42\% de estas familias perciben un ambiente armonioso con teleeducación y teletrabajo, y solo el $21 \%$ dice encontrarse en un contexto estresante, los padres desconocen las plataformas en las que sus hijos reciben clases y envían tareas porque no ejercen un acompañamiento intensivo en la educación de sus hijos como cuando se encuentran en niveles inferiores. En estas familias más que el uso de la tecnología en la educación, pueden tener inconvenientes con el trabajo virtual, la economía familiar incluso con pérdidas familiares y de conocidos. (Tabla 8).

Brechas digitales entre pares al relacionar los grupos de estudio se puede argumentar que existen brechas tecnológicas entre pares, los docentes del grupo 1 presentan un menor nivel de manejo y familiaridad con herramientas educativas virtuales en referencia con los docentes del grupo 2, sin embargo la realidad es que los centros educativos se encontraban apenas adoptando las TICS para mejorar el proceso de enseñanza, dinamizar el currículo escolar, la educación en todos los niveles y no solo a nivel nacional sino del país no se encontraba preparada para afrontar un cambio tan brusco e intempestivo por ello la adopción de la modalidad virtual representó un reto teniendo que afrontar múltiples obstáculos para los docentes, autoridades y estudiantes; los estudiantes del grupo 1 han sido los más afectados en cuanto a la modalidad virtual por la falta de recursos y medios tecnológicos así como el conocimiento y facilidad al momento de utilizar tecnología, puede ser debido al rango de edad, a pesar de encontrarnos en tiempos donde se afirma que el ser humano maneja dispositivos como celulares móviles y tecnología desde edades cada vez más tempranas, lo cierto es que esto genera dependencia y aunque puedan manejar redes sociales y juegos en línea, cuando 
se relaciona con cambiar totalmente un ambiente como es la educación presencial al medio virtual surgen inconvenientes que truncan el proceso, requiriendo más tiempo, capacitación, incluso actitud para que se logre el cometido que es educar con mayor o por lo menos igual calidad que en un aula física, ofreciendo las mismas oportunidades, un aspecto importante es que con esta modalidad la gratuidad educativa se ve comprometida si bien es cierto no pagan una pensión, pero en este sentido la conectividad y los dispositivos que son el medio principal para acceder a la teleeducación, corre por cuenta de las familias que en una situación de emergencia sanitaria con la pérdida de vidas y fuentes de ingresos se encuentran en una situación desfavorable.

Brecha digital entre grupos de estudio al relacionar los grupos de estudio resalta la desigualdad en las capacidades entre docentes, estudiantes y familias; sin duda mientras los docentes y estudiantes mediante sus medios han tratado de dar continuidad al proceso educativo, existen aún quienes no logran adaptarse al contexto. Por un lado tenemos a un grupo de docentes en el grupo 1 con grandes dificultades para preparar sus clases y herramientas metodológicas en el ambiente virtual, en el grupo 2 un poco más familiarizado pero no exento de problemas, en el otro extremo están los estudiantes de igual forma desorientados totalmente en un inicio, sin tener los recursos adecuados para tomar una educación virtual, al no poder preparar una clase de forma óptima repercute en la calidad del material presentado a los estudiantes, quienes no reciben o procesan de forma clara la información, quedando con vacíos, dudas y confusiones que le hacen difícil cumplir con las tareas encomendadas, las familias sin poder manejar las plataformas, sin tener una comunicación directa con el docente entra en desesperación al no poder ayudar al alumno a realizar su tarea cambia el contexto, pueden surgir cambios de humor, estrés y violencia doméstica lo que repercute en la integridad física y mental del alumno y su familia.

En este escenario no se puede detener la nueva realidad lo ideal es proseguir impulsando la construcción del aprendizaje significativo con la interacción paulatina de ambientes virtuales, que faciliten la conexión, comunicación y transferencia de conocimientos. 
Tabla 8. Resultado Familias Grupo 1 y 2

\begin{tabular}{|c|c|c|c|c|c|c|c|c|c|c|c|c|}
\hline \multirow{3}{*}{$\begin{array}{lc}\text { Estudiantes } \\
\text { Familias }\end{array}$} & \multicolumn{6}{|c|}{ Grupo 1} & \multicolumn{6}{|c|}{ Grupo 2} \\
\hline & \multicolumn{2}{|c|}{1 a 2} & \multicolumn{2}{|c|}{2 a 3} & \multicolumn{2}{|c|}{$(+)$ a 3} & \multicolumn{2}{|c|}{1 a 3} & \multicolumn{2}{|c|}{ 3a 5} & \multicolumn{2}{|c|}{$(+)$ a 5} \\
\hline & 27 & $10 \%$ & 53 & $20 \%$ & 187 & $70 \%$ & 85 & $32 \%$ & 24 & $9 \%$ & 158 & $59 \%$ \\
\hline \multirow[t]{2}{*}{ Cuántos de los miembros estudian } & 99 & $37 \%$ & 128 & $48 \%$ & 40 & $15 \%$ & 83 & $31 \%$ & 51 & $19 \%$ & 134 & $50 \%$ \\
\hline & \multicolumn{2}{|c|}{$\mathrm{Si}$} & \multicolumn{2}{|c|}{ No } & \multicolumn{2}{|c|}{ No Aplica } & \multicolumn{2}{|c|}{$\mathrm{Si}$} & \multicolumn{2}{|c|}{ No } & \multicolumn{2}{|c|}{ No Aplica } \\
\hline Disponía de computador, tablet antes de la pandemia & 53 & $20 \%$ & 214 & $80 \%$ & 0 & $0 \%$ & 107 & $40 \%$ & 160 & $60 \%$ & 0 & $0 \%$ \\
\hline \multirow[t]{2}{*}{ Disponía de conectividad a internet en su hogar antes de la pandemia } & 48 & $18 \%$ & 219 & $82 \%$ & 0 & $0 \%$ & 83 & $31 \%$ & 184 & $69 \%$ & 0 & $0 \%$ \\
\hline & \multicolumn{2}{|c|}{1 a 2} & \multicolumn{2}{|c|}{$(+)$ de 3} & \multicolumn{2}{|c|}{ No Aplica } & \multicolumn{2}{|c|}{1 a 2} & \multicolumn{2}{|c|}{$(+)$ de 3} & \multicolumn{2}{|c|}{ No Aplica } \\
\hline \multirow[t]{2}{*}{ Cuántos computadores dispone en su hogar } & 48 & $18 \%$ & 5 & $2 \%$ & 214 & $80 \%$ & 80 & $30 \%$ & 27 & $10 \%$ & 160 & $60 \%$ \\
\hline & \multicolumn{2}{|c|}{$\mathrm{Si}$} & \multicolumn{2}{|c|}{ No } & \multicolumn{2}{|c|}{ No Aplica } & \multicolumn{2}{|c|}{$\mathrm{Si}$} & \multicolumn{2}{|c|}{ No } & \multicolumn{2}{|c|}{ No Aplica } \\
\hline Conoce el manejo de la plataforma en la que su hijo/s sube sus tareas & 53 & $20 \%$ & 214 & $80 \%$ & 0 & $0 \%$ & 240 & $90 \%$ & 27 & $10 \%$ & 0 & $0 \%$ \\
\hline \multirow[t]{2}{*}{ Conoce el manejo de la aplicación en la que su hijo/s recibe clases } & 51 & $19 \%$ & 216 & $81 \%$ & 0 & $0 \%$ & 256 & $96 \%$ & 11 & $4 \%$ & 0 & $0 \%$ \\
\hline & & & & & & & & & & & & \\
\hline El control del cumplimiento de su hijo/s en la educación virtual es & 203 & $76 \%$ & 27 & $10 \%$ & 37 & $14 \%$ & 134 & $50 \%$ & 80 & $30 \%$ & 53 & $20 \%$ \\
\hline Las calificaciones de su hijo en la educación virtual son & 160 & $60 \%$ & 27 & $10 \%$ & 80 & $30 \%$ & 80 & $30 \%$ & 40 & $15 \%$ & 147 & $55 \%$ \\
\hline La asistencia a reuniones virtuales de padres de familia & 166 & $62 \%$ & 85 & $32 \%$ & 16 & $6 \%$ & 112 & $42 \%$ & 13 & $5 \%$ & 142 & $53 \%$ \\
\hline La comunicación con los docentes en medios virtuales es & 214 & $80 \%$ & 45 & $17 \%$ & 8 & $3 \%$ & 53 & $20 \%$ & 11 & $4 \%$ & 203 & $76 \%$ \\
\hline & & & & & & & & & & & & \\
\hline El uso de aplicaciones y dispositivos digitales le resulta & 37 & $14 \%$ & 67 & $25 \%$ & 163 & $61 \%$ & 83 & $31 \%$ & 134 & $50 \%$ & 51 & $19 \%$ \\
\hline & & & $(+) \mathrm{C}$ & diente & $\begin{array}{r}\text { Re } \\
\text { Des }\end{array}$ & $\begin{array}{l}\text { lde / } \\
\text { ligado }\end{array}$ & & & $(+) \mathrm{C}$ & diente & $\begin{array}{r}\operatorname{Re} \\
\text { Dese }\end{array}$ & $\begin{array}{l}\text { de / } \\
\text { gado }\end{array}$ \\
\hline El comportamiento de sus hijo/s con teleeducación ha sido & 37 & $14 \%$ & 16 & $6 \%$ & 184 & $69 \%$ & 147 & $55 \%$ & 40 & $15 \%$ & 80 & $30 \%$ \\
\hline & Ar & ioso & & & Inso & rtable & Arm & loso & & & Inso & table \\
\hline El ambiente en su hogar con clases virtuales y teletrabajo le resulta & 21 & $8 \%$ & 67 & $25 \%$ & 179 & $67 \%$ & 112 & $42 \%$ & 99 & $37 \%$ & 56 & $21 \%$ \\
\hline
\end{tabular}

Nota: Elaboración propia de los autores. 


\section{CONCLUSIONES}

Sin duda ante el contexto de una pandemia mundial, la teleeducación, así como el teletrabajo es la medida adecuada para frenar la propagación del virus y garantizar la integridad de docentes, estudiantes, familias y la sociedad en general; pero no basta con decretar mediante leyes y disposiciones, para que se logren los objetivos es necesario reducir las barreras de organización y capacitación, así como desigualdades entre involucrados.

En el estudio realizado se logró obtener una visión panorámica de los actores de las comunidades educativas mediante dos grupos el primero de nivel básico/medio y el segundo de nivel superior pertenecientes a la ciudad de Latacunga, resaltando la brecha tecnológica existente entre los grupos de estudio, lo que ha generado perspectivas negativas en los estudiantes, docentes y familias a quienes les ha costado adaptarse a los medios virtuales y en gran proporción la escases de recursos de los hogares, no se debe desvalorizar la educación presencial que ha existido desde siempre, pero es importante que se acoja las nuevas formas de enseñanza, lo que ha fallado es la premura con que cambió la realidad pero, a largo plazo la educación en línea puede revolucionar la enseñanza, ya se ha dado el primer paso lo siguiente es perfeccionar las metodologías y ritmos de enseñanza para enriquecer y mejorar los logros pedagógicos.

El grupo 1 perteneciente a educación básica y media es el más afectado en cuánto a la modalidad virtual de educación, es el más renuente a adaptarse a ambientes tecnológicos, los docentes, estudiantes y familias de este grupo presentan una mayor desigualdad, menor disponibilidad de recursos $y$ un nivel bajo de competencias virtuales, los docentes antes de la pandemia manejaban solo programas básicos que les permitían cumplir con la programación curricular en modalidad presencial, teniendo dificultades para generar espacios virtuales de aprendizaje colaborativo y participativo.

El grupo 2 al pertenecer al nivel de educación superior se encuentra más familiarizado con plataformas educativas en línea, se adaptaron a la teleeducación en seis meses promedio y las familias de este grupo no muestran mayor alteración emocional ni funcional, sin embargo, coinciden en un rendimiento más bajo por parte de los estudiantes, disminución de la calidad educativa asociada a una mayor carga y estrés laboral.

\section{REFERENCIAS}

Bedoya Dorado, C., Murillo Vargas, G., y González Campo, C. H. (27 de 06 de 2021). GESTIÓN UNIVERSITARIA EN TIEMPOS DE PANDEMIA POR COVID -19: ANÁLISIS DEL SECTOR DE LA EDUCACIÓN SUPERIOR EN COLOMBIA . Recuperado el 18 de 08 de 2021, de SciELO : http://www.scielo.org.co/ scielo.php?script $=$ sci_arttext \&pid $=S 0123$ 59232021000200251\&lang=es

Bonilla Guachamín, J. A. (25 de 05 de 2020). LAS DOS CARAS DE LA EDUCACIÓN EN EL COVID-19. Recuperado el 14 de 08 de 2021, de Universidad Tecnológica Indoamérica: http:// cienciamerica.uti.edu.ec/openjournal/index. $\mathrm{php/uti/article/view/294/462}$

Carreño, M. (06 de 10 de 2020). TRAS LA PANDEMIA, EN ECUADOR BAJÓ LA CALIDAD DE LA EDUCACIÓN . Recuperado el 14 de 08 de 2021, de Pastoral Social Cáritas Ecuador : https://www.caritasecuador. org/2020/10/pandemia-calidad-educacion/

Castro, M. (29 de 07 de 2020). TIEMPOS DE PANDEMIA: CÓMO ES EL ACCESO A LA EDUCACIÓN EN ECUADOR MIENTRAS EL COVID-19 AFECTA LA REGIÓN . 
Recuperado el 14 de 08 de 2021, de National Geographic: https://www.nationalgeographicla. com/fotografia/2020/07/tiempos-de-pandemiaacceso-a-la-educacion-en-ecuador

Dirección Distrital 05D01 Latacunga-Educación. (12 de 2020). INFORMA DE RENDICIÓN DE CUENTAS COORDINACIÓN ZONAL DE EDUCACIÓN ZONA 3 ENERO-DICIEMBRE 2020. Recuperado el 14 de 05 de 2021, de MINISTERIO DE EDUCACIÓN : https:// educacion.gob.ec/wp-content/uploads/ downloads/2021/05/05D01.pdf

Lozada Martinez, I. D., y Aristizabal Carmona, B. S. (01 de 04 de 2021). SIMULADORES VIRTUALES COMO HERRAMIENTAS FUNDAMENTALES PARA LA EDUCACIÓN MÉDICA CLINICA EN TIEMPOS DE COVID-19. Recuperado el 18 de 08 de 2021, de SciELO: http://scielo.sld.cu/ scielo.php?script $=$ sci_arttext $\&$ pid $=$ S0864 21412021000100003\&lang=es

Pérez Constante, M.B., y Tramallino, C. P. (01 de 07 de 2020). LAS CONSECUENCIAS EDUCATIVAS $Y$ EL DESARROLLO DEL DOCENTE A CAUSA DEL USO DE LAS TIC'S EN LAS REFORMAS Y TIPOS DE APRENDIZAJE EN TIEMPOS DEL COVID-19. Recuperado el 14 de 08 de 2021, de Magazine de las Ciencias: Revista de Investigación e Innovación : https:// revistas.utb.edu.ec/index.php/magazine/article/ view/900/681
Ramos Duarte, S., Bouzó, A., y Santiviago, C. (01 de 06 de 2021). ESTRATEGIA DE APOYO ESTUDIANTIL EN TIEMPOS DE PANDEMIA. Recuperado el 18 de 08 de 2021, de SciELO: http://www.scielo.edu.uy/ scielo.php?script $=$ sci_arttext \&pid=S230101262021000100035\&lang=es

SENESCYT Secretaria de Educación Superior. (2020). Sistema Integral de Información de Educación Superior (SIIES). Recuperado el 14 de 05 de 2021, de https://siau.senescyt.gob.ec/ estadisticas-de-educacion-superior-cienciatecnologia-e-innovacion/?doing_wp_cron $=162$ 1020771.2511219978332519531250

UNICEF Fondos para las Naciones Unidas. (19 de 11 de 2020). UNICEF PIDE EVITAR DAÑOS IRREVERSIBLES EN EDUCACIÓN, SALUD, NUTRICIÓN Y BIENESTAR DE LOS NIÑOS Y LAS NIÑAS A CAUSA DE LA PANDEMIA. Recuperado el 14 de 08 de 2021, de UNICEF: https://www.unicef.org/ecuador/comunicadosprensa/unicef-pide-evitar-da\%C3\%B 1osirreversibles-en-educaci\%C3\%B3n-saludnutrici\%C3\%B3n-y-bienestar

UNIR, L. (08 de 03 de 2021). INNOVACIÓN TECNOLÓGICA EN LA EDUCACIÓN: TENDENCIAS, HERRAMIENTAS Y MEJORAS EJEMPLOS. Recuperado el 14 de 08 de 2021, de méxico.unir.ec: https://mexico. unir.net/vive-unir/innovacion-tecnologicaeducacion/ 